\title{
Fluorination of Donor-Acceptor Copolymer Active Layers Enhances Charge Mobilities in Thin-Film Transistors
}

\author{
Brandon H. Smith, ${ }^{\dagger}$ Qianqian Zhang, ${ }^{\ddagger}$ Mary Allison Kelly, ${ }^{\ddagger}$ Joshua H. Litofsky, $^{\dagger}$ Dinesh Kumar, ${ }^{\S}$ \\ Alexander Hexemer, ${ }^{\S}$ Wei You, ${ }^{\ddagger}$ and Enrique D. Gomez ${ }^{*},,, \| \Subset$ \\ ${ }^{\dagger}$ Department of Chemical Engineering and "Materials Research Institute, The Pennsylvania State University, University Park, \\ Pennsylvania 16802, United States \\ ${ }^{\ddagger}$ Department of Chemistry, University of North Carolina, Chapel Hill, North Carolina 27514, United States \\ ${ }^{\S}$ Advanced Light Source, Lawrence Berkeley National Laboratory, Berkeley, California 94720, United States
}

ABSTRACT: Several recent reports have demonstrated that fluorinated analogues of donor/acceptor copolymers surpass nonfluorinated counterparts in terms of performance in electronic devices. Using a copolymer series consisting of fluorinated, partially fluorinated, and nonfluorinated benzotriazole, we confirm that the addition of fluorine substituents beneficially impacts charge transport in polymer semiconductors. Transistor measurements demonstrated a factor of 5 increase in carrier mobilities with the degree of fluorination of the backbone. Furthermore, grazing-incidence X-ray diffraction data indicates progressively closer packing between the conjugated cores and an overall greater amount of $\pi$-stacking in the fluorinated materials. It is likely that attractive interactions between the electronrich donor and fluorinated electron-deficient acceptor units induce

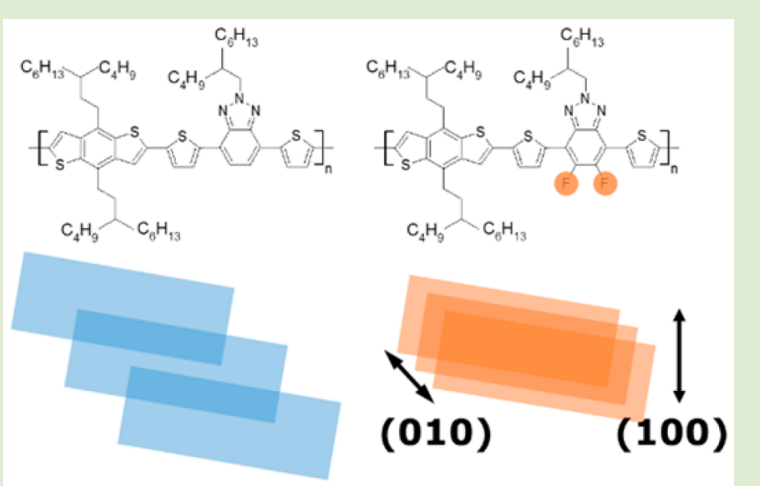
very tightly stacking crystallites, which reduce the energetic barrier for charge hopping. In addition, a change in crystallite orientation was observed from primarily edge-on without fluorine substituents to mostly face-on with fluorinated benzotriazole.

$\mathrm{W}$ ell-designed materials based on the widely adopted donor/acceptor copolymer model, whereby a weak donor group is coupled with a strong acceptor group, often exhibit excellent electronic properties. ${ }^{1}$ Furthermore, fluorinated analogues of such copolymers often surpass their nonfluorinated counterparts in terms of performance in devices. $^{2}$ As such, solar cells incorporating fluorinated polymers in the active layer have claimed three of the top eight slots in terms of power-conversion efficiency, according to a recent review. ${ }^{3-5}$ In each case, the fluorinated variant consisted of a benzo $\left[1,2-b: 4,5-b^{\prime}\right]$ dithiophene $(\mathrm{BnDT})$ donor copolymerized with an electron-deficient acceptor possessing a strongly electron withdrawing fluorine substituent. Specific examples of the fluorinated acceptor moieties include thienothiophene (TT), benzotriazole (TAZ), and benzothiadiazole (BT) (Tables S1 and S2 of the Supporting Information). ${ }^{6-8}$ Copolymers containing a fluorinated $\mathrm{BnDT}$ donor have also been explored, but despite a significant reduction in both the HOMO and LUMO values, photovoltaic performance is much lower than when fluorinated acceptor units are incorporated in the active layer (Table S3 of the Supporting Information). ${ }^{9}$

In search of a mechanistic description of the role of fluorine substitution, multiple efforts have probed various optoelectronic properties of blends of conjugated polymers mixed with fullerene, such as charge transport efficacy, morphology, and charge dissociation or recombination. ${ }^{10-12}$ Studies examining
PTB7, PBnDT-DTffBT, PBnDT-FTAZ, and similar fluorinated materials have provided many practical insights into the behavior and interactions associated with the fluorine substituents. ${ }^{13,14}$ Nevertheless, it appears that a consensus has yet to be achieved, as the observed fluorine-induced enhancements have been attributed to a variety of distinct factors. The four primary reasons put forth include improved morphology, decreased charge recombination, improved packing and orientation with the addition of fluorine, and improved hole mobility, as outlined with more detail in the Supporting Information.

While the findings of prior studies provide substantial illumination and suggest that the hole mobility of the PBnDTTAZ system is the most critical parameter altered by fluorine substituents, key analyses remain incomplete. For example, thin-film transistors (TFTs) provide a method for directly observing the charge mobility of semiconductors, yet TFT measurements have not been reported for PBnDT-FTAZ active layers. Mobilities presented in prior reports were extracted from space charge limited current (SCLC) measurements with diodes, which are not always a reliable indication of the true

Received: September 14, 2017

Accepted: September 26, 2017

Published: October 2, 2017 
charge transport potential of organic semiconductors. ${ }^{15,16}$ In addition, the improved mobility was correlated with enhancements in the $\pi$-stacking observed from GIWAXS measurements of polymer/fullerene films, although the presence of a fullerene reflection near the location of the polymer $\pi$-stacking peak convolutes the results. Consequently, neat polymers must be analyzed to more accurately differentiate the changes occurring as the extent of fluorination increases.

In this Letter, we investigate the effect of substituting fluorine groups onto the high mobility conjugated donor/acceptor PBnDT-(X)TAZ copolymer series, shown in Figure 1, as a

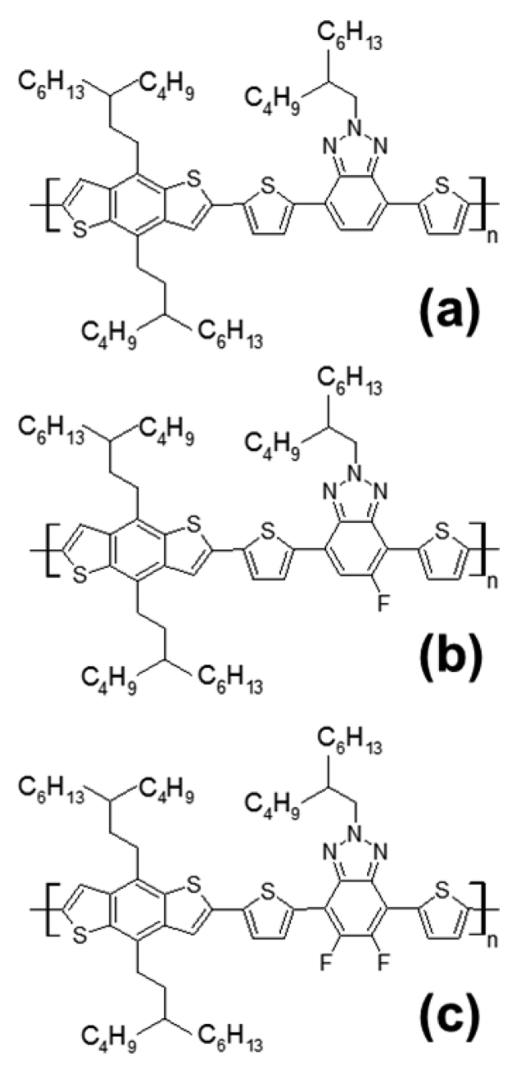

Figure 1. Chemical structure of the fluorinated PBnDT-TAZ copolymer series including (a) PBnDT-HTAZ, (b) PBnDT-mFTAZ, and (c) PBnDT-FTAZ.

model system for probing the origin of the "fluorine effect". Neat nonfluorinated (PBnDT-HTAZ), monofluorinated (PBnDT-mFTAZ), and difluorinated (PBnDT-FTAZ) materials were examined, as opposed to the polymer/fullerene blends studied previously. In contrast to prior work, the partially fluorinated copolymer consisted entirely of monofluorinated PBnDT-TAZ rather than a specified ratio between PBnDTHTAZ and PBnDT-FTAZ units in a single chain. ${ }^{2}$ The similarity between each copolymer variant allowed us to systematically survey the change in hole mobility while holding all other parameters constant. Hence, the goal of this work was to address whether the addition of fluorine influences the charge transport properties of polymer semiconductors. We compared the charge mobility of the neat copolymers with TFTs and probed the structure with GIWAXS, confirming a structural origin of mobility enhancement.

TFTs were fabricated by casting from PBnDT-HTAZ, PBnDT-mFTAZ, and PBnDT-FTAZ solutions onto $\mathrm{Si} / \mathrm{SiO}_{2} /$ $\mathrm{Au}$ substrates treated with various self-assembled monolayers.
Device fabrication details can be found elsewhere. ${ }^{17}$ The number-averaged molecular weights of all polymers are above $50 \mathrm{~kg} / \mathrm{mol}$ (Figure S1 and Table S4 of the Supporting Information), such that the differences in molecular weight should not significantly affect charge mobilities. ${ }^{18}$ As shown in Figures S3-S5 and Table S5 of the Supporting Information, octadecyltrichlorosilane (ODTS)-treated substrates maximized TFT performance. Optimization of thermal annealing conditions led to annealing of devices at $150{ }^{\circ} \mathrm{C}$.

Representative output and transfer curves for devices fabricated from each copolymer variant are shown in Figure 2. No signatures of contact barriers are apparent in the output
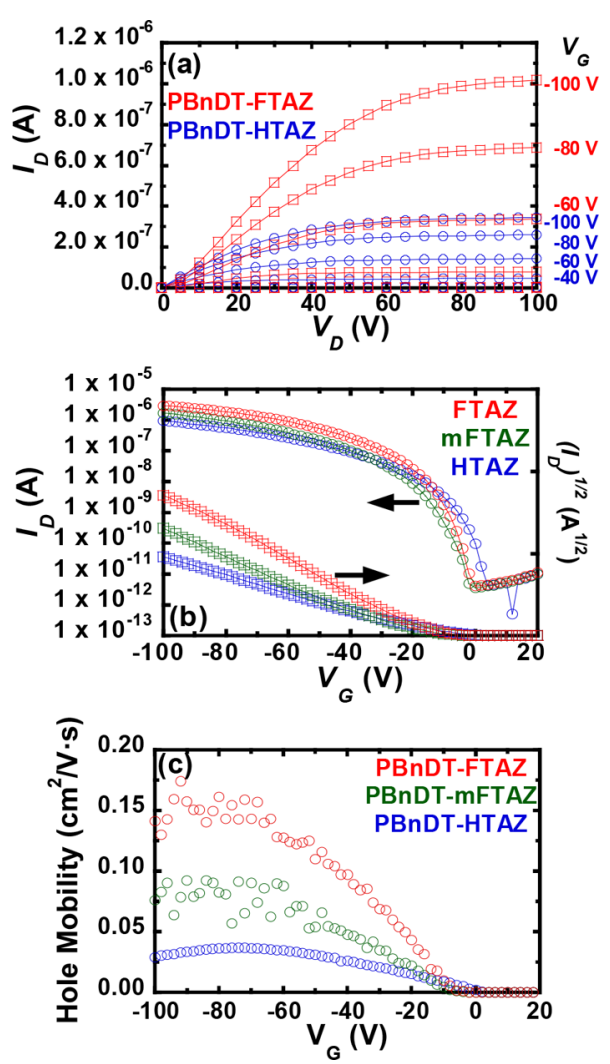

Figure 2. (a) Output, (b) transfer, and (c) mobility plots for representative TFTs prepared from PBnDT-HTAZ, PBnDT-mFTAZ, and PBnDT-FTAZ copolymers. Channel length and width dimensions were 320 and $220 \mu \mathrm{m}$, respectively. The dielectric layer was $300 \mathrm{~nm}$ of $\mathrm{SiO}_{2}$, and the source-drain bias was $100 \mathrm{~V}$.

curves of Figure 2a. ${ }^{19,20}$ The source-drain current is highest for devices with PBnDT-FTAZ active layers, leading to higher charge mobilities. Recent work suggests that contact problems can lead to overestimation of charge mobilities from TFTs, which is manifested as a peak in the mobility with gate voltage. ${ }^{19,20}$ Hole mobilities versus gate voltage for devices with the three polymers as the active layer are shown in Figure $2 \mathrm{c}$. Mobilities are roughly independent of gate voltage for a large range, as expected for devices with minimal contact problems.

Figure 3 shows average threshold voltages, ON/OFF ratios, and charge mobilities, where annealing after 3 or $11 \mathrm{~h}$ leads to similar results. All threshold voltages are within about $5 \mathrm{~V}$ of zero, and ON/OFF ratios are in the $10^{5}$ to $10^{6}$ range. Although hole mobilities are roughly constant for as cast samples, mobilities improve by approximately a factor of 5 from 0.03 $\mathrm{cm}^{2} / \mathrm{V} \cdot \mathrm{s}$ to $0.08 \mathrm{~cm}^{2} / \mathrm{V} \cdot \mathrm{s}$ and $0.14 \mathrm{~cm}^{2} / \mathrm{V} \cdot \mathrm{s}$, increasing with the 

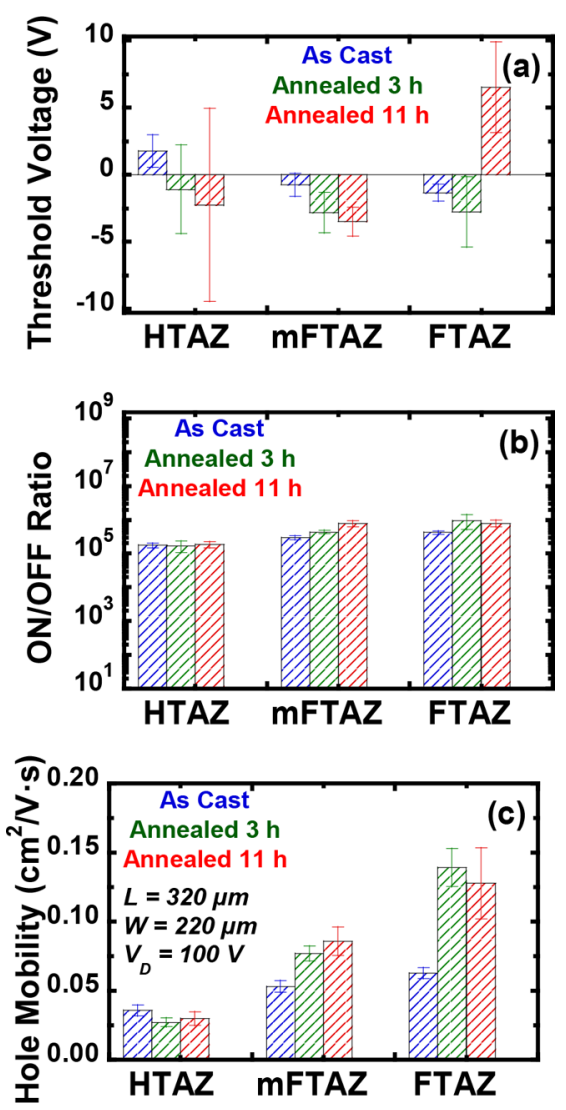

Figure 3. (a) Average threshold voltages, (b) average ON/OFF ratios, and (c) average charge mobilities for devices comprised of polymers with various degrees of fluorine substitution in the active layer. Errors bars are the standard deviation of multiple measurements $(n \geq 10)$.

number of fluorine substituents incorporated onto the conjugated polymer backbone (Figure $3 \mathrm{c}$ ).

Comparing our mobility values with those reported from SCLC diode measurements reveals some limitations of that method. While the trends and specific SCLC hole mobility for PBnDT-FTAZ $\left(\sim 1 \times 10^{-3} \mathrm{~cm}^{2} / \mathrm{V} \cdot \mathrm{s}\right)$ are in close agreement with the average TFT mobility extracted from measurements on untreated $\mathrm{SiO}_{2}\left(\sim 3 \times 10^{-3} \mathrm{~cm}^{2} / \mathrm{V} \cdot \mathrm{s}\right)$, we propose that TFT characterization is important to fully characterize the in-plane charge transport efficacy. We have also examined the mobility of two other fluorinated donor-acceptor copolymers, namely, PTB7 and PBDTT-FTTE (Figure S6 of the Supporting Information). These monofluorinated materials demonstrate exceptional performance approaching that of mFTAZ, as illustrated in Figures S7 and S8 of the Supporting Information.

Charge mobilities near $0.1 \mathrm{~cm}^{2} /(\mathrm{V} \cdot \mathrm{s})$ help explain the remarkable performance of PBnDT-FTAZ in organic photovoltaics. Previous work has demonstrated power conversion efficiencies near $7 \%$ with active layers of about $300 \mathrm{~nm}$ in thickness. ${ }^{2,5,21}$ We propose that the high hole mobility of PBnDT-FTAZ enables thick active layers with fill factors in excess of 0.7 to allow for efficient light harvesting without significant losses due to charge extraction problems.

To ascertain the underlying cause for the increase in TFT mobilities with added fluorine in the active layer, we examined the crystalline structure of the three polymers shown in Figure 1 using X-ray diffraction experiments. PBnDT-HTAZ, PBnDTmFTAZ, and PBnDT-FTAZ films were prepared on untreated and ODTS-treated silicon wafers for grazing-incidence wide- angle X-ray scattering (GIWAXS) measurements (Figure S9 of the Supporting Information and Figure 4). Data were acquired
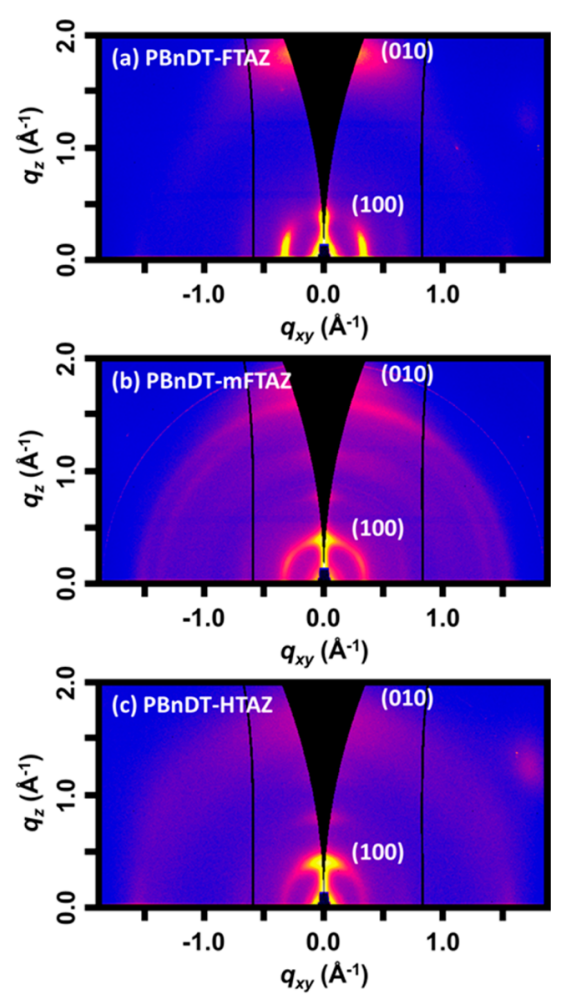

Figure 4. GIWAXS images for (a) PBnDT-FTAZ, (b) PBnDTmFTAZ, and (c) PBnDT-HTAZ films on ODTS-treated silicon. Samples were annealed $3 \mathrm{~h}$ at $150{ }^{\circ} \mathrm{C}$.

for films as cast and after annealing for $3 \mathrm{~h}$ at $150{ }^{\circ} \mathrm{C}$. Although differential scanning calorimetry data shows no thermal transitions in the range of 50 to $250{ }^{\circ} \mathrm{C}$ (data not shown), comparing GIWAXS data from annealed (Figure 4) and not annealed (Figure S10 of the Supporting Information) films suggests that annealing at $150{ }^{\circ} \mathrm{C}$ induces cold crystallization.

For each polymer, the integrated profiles extracted from the two-dimensional detector images in Figure 4 are displayed in Figures 5 and S11 in the Supporting Information. The (100) and (010) peaks at $q \sim 0.3-0.4 \AA^{-1}$ and $q \sim 1.5-1.7 \AA^{-1}$ correspond to backbone-side chain lamellar and $\pi-\pi$ stacking, respectively, but it is apparent from the detector images and line profiles that the overall crystallinity is low for all three copolymers. The absence of higher order reflections and the presence of broad peaks indicate a high degree of disorder and short coherence lengths, which is consistent with previous reports $^{22}$ and implies that crystallinity and long-range order will have only a minor influence on charge transport. Results obtained on untreated and SAM-treated substrates were similar, as shown in Figure $S 9$ of the Supporting Information, but slightly sharper peaks observed from ODTS treated samples indicate that the polymers were more uniformly oriented with the silane monolayer. Reorientation of PBnDT-FTAZ crystallites from edge-on to face-on with respect to the substrate has been reported following solvent annealing. ${ }^{10}$ A comparison between our as cast and thermally annealed films (Figure S10 of the Supporting Information and Figure 4), however, do not reveal any obvious change in orientation.

In contrast, a transition from primarily edge-on to face-on orientation occurs gradually as the extent of fluorination 

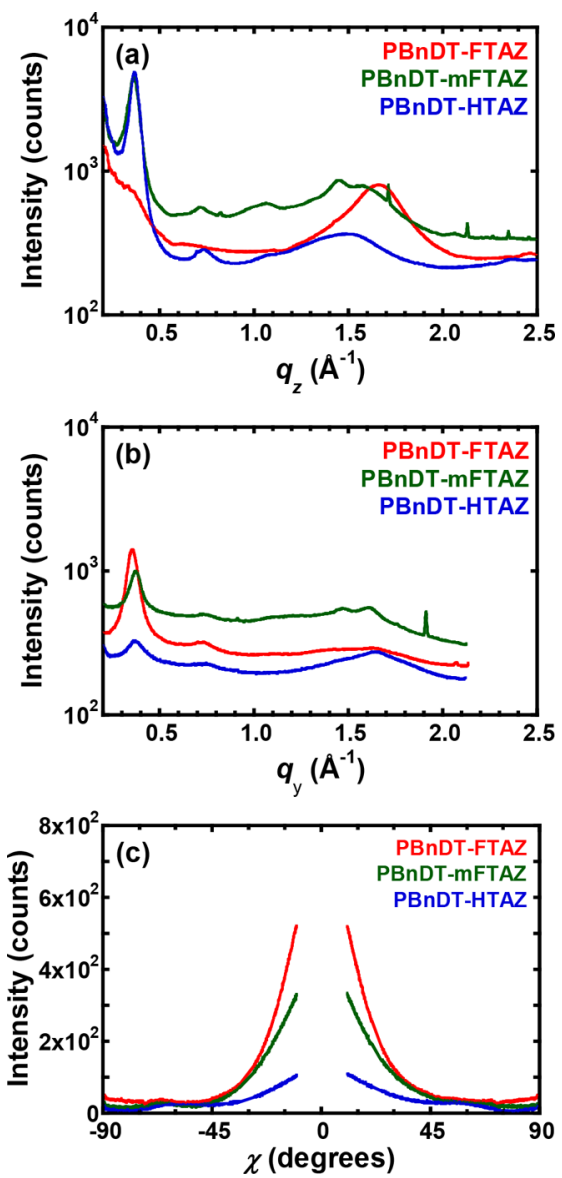

Figure 5. (a) Out-of-plane sector cuts $\left( \pm 10^{\circ}\right.$ from vertical $)$ and (b) inplane sector cuts $\left(+20^{\circ}\right.$ from horizontal) of GIWAXS data for PBnDTHTAZ, PBnDT-mFTAZ, and PBnDT-FTAZ films on ODTS-treated substrates. (b) GIWAXS intensities vs polar angle $\chi$ at the (010) peak. All samples were annealed at $150{ }^{\circ} \mathrm{C}$ for $3 \mathrm{~h}$.

changes when ODTS treated substrates are used (Figure 4). Nevertheless, all polymers exhibit a significant population of face-on crystals when cast and annealed on bare Si substrates (Figure S9 of the Supporting Information). We hypothesize that the low surface energy of ODTS $\left(28.1 \mathrm{~mJ} / \mathrm{m}^{2}\right)^{23}$ promotes edge-on texturing of the crystallites when compared to high energy substrates (bare $\left.\mathrm{Si}, 61.4 \mathrm{~mJ} / \mathrm{m}^{2}\right)^{23}$ due to interactions with the alkyl side chains, but $\mathrm{F}-\mathrm{H}$ interactions between the polymer and ODTS can also play a role to promote face-on orientations for fluorinated materials. Interestingly, although older work has hypothesized that edge-on orientations can promote in-plane charge transport, ${ }^{24}$ we observe the highest mobilities for devices where the active layer is mostly face-on (PBnDT-FTAZ devices). Furthermore, recent evidence suggests that transport through conjugated polymers is quasi one-dimensional, such that both edge-on and face-on crystal texturing can lead to efficient charge transport. ${ }^{25,26}$ Thus, we speculate that further work is needed to explore whether edgeon crystallites in the active layer always enhance charge transport, or whether developing strategies to promote edge-on stacking in PBnDT-FTAZ will enhance charge mobilities.

A close scrutiny of the out-of-plane line profiles reveals that the position of the (010) peak shifts toward higher $q$ for the fluorinated analogues. The $\pi-\pi$ distances decrease from roughly $4.09 \AA$ for PBnDT-HTAZ to $3.71 \AA$ in PBnDTFTAZ films. The smaller $\pi$-stacking distance is consistent with the tighter packing observed in fluorine-substituted aromatic moieties. ${ }^{27}$ The PBnDT-mFTAZ film also exhibits a reflection near $1.49 \AA^{-1}$, which is apparent in PBnDT-HTAZ for films cast on ODTS but not annealed (Figure S10 of the Supporting Information). Thus, this peak is likely due to the (001) reflection or a mixed index reflection and not $\pi$-stacking. The azimuthally integrated (010) peak intensities, provided in Figure $5 \mathrm{~b}$, also reveal a significant rise in the amount of $\pi$ stacking with fluorine content, as evidenced by the vastly greater intensity. As such, we propose that the reduced distance between rings and the increased amount of $\pi$-stacking in the active layer explain the increase in charge mobilities extracted from devices.

In conclusion, our work has characterized the charge mobility of a series of nonfluorinated and fluorinated PBnDT-TAZ copolymers in TFTs. Mono- and difluorinated analogues of PBnDT-HTAZ allowed the effect of fluorine substitution to be decoupled from ancillary influences and revealed that the fluorine atoms improved charge transport within the copolymer. An optimization study investigating the effects of different types of SAM treatments and thermal annealing conditions was conducted, which demonstrated that ODTS surface treatments maximized charge transport. Average hole mobilities of 0.08 and $0.14 \mathrm{~cm}^{2} / \mathrm{V} \cdot \mathrm{s}$, exceeding that of $\mathrm{P}_{3} \mathrm{HT}^{17,28-30}$ and approaching that of PBTTT, ${ }^{31,32}$ are achieved with the mono- and dual-fluorinated PBnDT-TAZ despite the low degree of long-range order in both copolymers.

None of the copolymers were highly crystalline, but probing the structure with GIWAXS shows that the excellent charge mobility originates from decreased $\pi$-stacking distances and an increase in the overall amount of $\pi$-stacking induced by the fluorine substituents. As such, the observed $0.38 \AA$ decrease in the stacking distance from $4.09 \AA$ without fluorination to $3.71 \AA$ with fluorination served to boost performance in devices by roughly a factor of 5. A change in the crystallite orientation to a face-on alignment was also noted upon fluorination, although we hypothesize that this most likely did not augment performance in TFTs. We propose that attractive interactions between electron-rich donor and fluorinated electron-deficient acceptor units yield very close $\pi$-stacking crystallites. The small diameter of the fluorine atom does not induce steric hindrances, thereby permitting tight packing. As such, PBnDT-FTAZ and similar fluorinated donor/acceptor materials offer a promising route for improving the charge mobility of organic devices.

\section{ASSOCIATED CONTENT}

\section{Supporting Information}

The Supporting Information is available free of charge on the ACS Publications website at DOI: 10.1021/acsmacrolett.7b00716.

Experimental details, GPC and UV-vis data, a brief review of relevant background work, results of a dielectric surface treatment optimization study, details regarding the TFT characterization of two other fluorinated high performance organic semiconductors, and additional GIWAXS data (PDF).

\section{AUTHOR INFORMATION}

\section{Corresponding Author}

*E-mail: edg12@psu.edu. 


\section{ORCID}

Qianqian Zhang: 0000-0002-8896-1708

Enrique D. Gomez: 0000-0001-8942-4480

Notes

The authors declare no competing financial interest.

\section{ACKNOWLEDGMENTS}

This work was funded by the Center for Flexible Electronics at the Pennsylvania State University and the Dow Chemical Company. The Advanced Light Source is an Office of Science user facility operated for the U.S. Department of Energy Office of Science by Lawrence Berkeley National Laboratory and supported by the U.S. Department of Energy under Contract No. DE-AC02-05CH11231.

\section{ABBREVIATIONS}

PBnDT-TAZ poly(benzodithiophene)-poly(benzotriazole) copolymer

GIWAXS grazing-incidence wide-angle X-ray diffraction

TFT thin-film transistor

$\mathrm{SiO}_{2} \quad$ silicon dioxide; $\mathrm{Au}$, gold

P3HT poly(3-hexylthiophene)

TCB 1,2,4-trichlorobenzene

SAM self-assembled monolayer

RPM revolutions per minute

HOMO highest occupied molecular orbital

LUMO lowest unoccupied molecular orbital

ODTS octadecyltrichlorosilane

SCLC space charge limited current

TT thienothiophene

TAZ benzotriazole

BT benzothiadiazole

MW weight-averaged molecular weight

D dispersity.

\section{REFERENCES}

(1) Zhou, H.; Yang, L.; Stoneking, S.; You, W. A weak donor-strong acceptor strategy to design ideal polymers for organic solar cells. ACS Appl. Mater. Interfaces 2010, 2 (5), 1377-1383.

(2) Li, W. T.; Abrecht, S.; Yang, L. Q.; Roland, S.; Tumbleston, J. R.; McAfee, T.; Yan, L.; Kelly, M. A.; Ade, H.; Neher, D.; You, W. Mobility-Controlled Performance of Thick Solar Cells Based on Fluorinated Copolymers. J. Am. Chem. Soc. 2014, 136 (44), 1556615576.

(3) Uy, R. L.; Price, S. C.; You, W. Structure-property optimizations in donor polymers via electronics, substituents, and side chains toward high efficiency solar cells. Macromol. Rapid Commun. 2012, 33 (14), $1162-1177$.

(4) Leclerc, N.; Chávez, P.; Ibraikulov, O.; Heiser, T.; Lévêque, P. Impact of Backbone Fluorination on $\pi$-Conjugated Polymers in Organic Photovoltaic Devices: A Review. Polymers 2016, 8 (11), 1-27.

(5) Xiao, S.; Zhang, Q; You, W. Molecular Engineering of Conjugated Polymers for Solar Cells: An Updated Report. Adv. Mater. 2017, 29, 1601391.

(6) Liang, Y.; Xu, Z.; Xia, J.; Tsai, S. T.; Wu, Y.; Li, G.; Ray, C.; Yu, L. For the bright future-bulk heterojunction polymer solar cells with power conversion efficiency of 7.4\%. Adv. Mater. 2010, 22 (20), E135-E138.

(7) Zhou, H.; Yang, L.; Stuart, A. C.; Price, S. C.; Liu, S.; You, W. Development of Fluorinated Benzothiadiazole as a Structural Unit for a Polymer Solar Cell of 7\% Efficiency. Angew. Chem. 2011, 123 (13), 3051-3054.

(8) Price, S. C.; Stuart, A. C.; Yang, L.; Zhou, H.; You, W. Fluorine substituted conjugated polymer of medium band gap yields $7 \%$ efficiency in polymer-fullerene solar cells. J. Am. Chem. Soc. 2011, 133 (12), 4625-4631.

(9) Son, H. J.; Wang, W.; Xu, T.; Liang, Y.; Wu, Y.; Li, G.; Yu, L. Synthesis of fluorinated polythienothiophene-co-benzodithiophenes and effect of fluorination on the photovoltaic properties. J. Am. Chem. Soc. 2011, 133 (6), 1885-1894.

(10) Tumbleston, J. R.; Stuart, A. C.; Gann, E.; You, W.; Ade, H. Fluorinated Polymer Yields High Organic Solar Cell Performance for a Wide Range of Morphologies. Adv. Funct. Mater. 2013, 23 (27), 3463-3470.

(11) Wang, N.; Chen, Z.; Wei, W.; Jiang, Z. H. Fluorinated Benzothiadiazole-Based Conjugated Polymers for High-Performance Polymer Solar Cells without Any Processing Additives or Posttreatments. J. Am. Chem. Soc. 2013, 135 (45), 17060-17068.

(12) Albrecht, S.; Janietz, S.; Schindler, W.; Frisch, J.; Kurpiers, J.; Kniepert, J.; Inal, S.; Pingel, P.; Fostiropoulos, K.; Koch, N.; Neher, D. Fluorinated Copolymer PCPDTBT with Enhanced Open-Circuit Voltage and Reduced Recombination for Highly Efficient Polymer Solar Cells. J. Am. Chem. Soc. 2012, 134 (36), 14932-14944.

(13) Stuart, A. C.; Tumbleston, J. R.; Zhou, H. X.; Li, W. T.; Liu, S. B.; Ade, H.; You, W. Fluorine Substituents Reduce Charge Recombination and Drive Structure and Morphology Development in Polymer Solar Cells. J. Am. Chem. Soc. 2013, 135 (5), 1806-1815.

(14) Son, H. J.; Wang, W.; Xu, T.; Liang, Y. Y.; Wu, Y. E.; Li, G.; Yu, L. P. Synthesis of Fluorinated Polythienothiophene-co-benzodithiophenes and Effect of Fluorination on the Photovoltaic Properties. J. Am. Chem. Soc. 2011, 133 (6), 1885-1894.

(15) Li, Y.; Clevenger, R. G.; Jin, L.; Kilway, K. V.; Peng, Z. SpinCoated Thin Films of Polycyclic Aromatic Hydrocarbons Exhibiting High SCLC Hole Mobilities. J. Phys. Chem. C 2016, 120 (2), 841-852.

(16) Tanase, C.; Meijer, E. J.; Blom, P. W.; De Leeuw, D. M. Unification of the hole transport in polymeric field-effect transistors and light-emitting diodes. Phys. Rev. Lett. 2003, 91 (21), 216601.

(17) Smith, B. H.; Clark, M. B.; Kuang, H.; Grieco, C.; Larsen, A. V.; Zhu, C.; Wang, C.; Hexemer, A.; Asbury, J. B.; Janik, M. J.; Gomez, E. D. Controlling Polymorphism in Poly(3-Hexylthiophene) through Addition of Ferrocene for Enhanced Charge Mobilities in Thin-Film Transistors. Adv. Funct. Mater. 2015, 25 (4), 542-551.

(18) Noriega, R.; Rivnay, J.; Vandewal, K.; Koch, F. P. V.; Stingelin, N.; Smith, P.; Toney, M. F.; Salleo, A. A general relationship between disorder, aggregation and charge transport in conjugated polymers. Nat. Mater. 2013, 12 (11), 1038-1044.

(19) Bittle, E. G.; Basham, J. I.; Jackson, T. N.; Jurchescu, O. D.; Gundlach, D. J. Mobility overestimation due to gated contacts in organic field-effect transistors. Nat. Commun. 2016, 7, 10908.

(20) Sirringhaus, H. 25th Anniversary Article: Organic Field-Effect Transistors: The Path Beyond Amorphous Silicon. Adv. Mater. 2014, 26 (9), 1319-1335.

(21) Price, S. C.; Stuart, A. C.; Yang, L. Q.; Zhou, H. X.; You, W. Fluorine Substituted Conjugated Polymer of Medium Band Gap Yields 7\% Efficiency in Polymer-Fullerene Solar Cells. J. Am. Chem. Soc. 2011, 133 (12), 4625-4631.

(22) DeLongchamp, D. M.; Kline, R. J.; Fischer, D. A.; Richter, L. J.; Toney, M. F. Molecular Characterization of Organic Electronic Films. Adv. Mater. 2011, 23 (3), 319-337.

(23) Lim, S. C.; Kim, S. H.; Lee, J. H.; Kim, M. K.; Kim, D. J.; Zyung, T. Surface-treatment effects on organic thin-film transistors. Synth. Met. 2005, 148 (1), 75-79.

(24) Sirringhaus, H.; Brown, P. J.; Friend, R. H.; Nielsen, M. M.; Bechgaard, K.; Langeveld-Voss, B. M. W.; Spiering, A. J. H.; Janssen, R. A. J.; Meijer, E. W.; Herwig, P.; de Leeuw, D. M. Two-dimensional charge transport in self-organized, high-mobility conjugated polymers. Nature 1999, 401 (6754), 685-688.

(25) Zhang, X. R.; Bronstein, H.; Kronemeijer, A. J.; Smith, J.; Kim, Y.; Kline, R. J.; Richter, L. J.; Anthopoulos, T. D.; Sirringhaus, H.; Song, K.; Heeney, M.; Zhang, W. M.; McCulloch, I.; DeLongchamp, D. M. Molecular origin of high field-effect mobility in an indacenodithiophene-benzothiadiazole copolymer. Nat. Commun. 2013, 4, 2238. 
(26) Guo, C.; Lee, Y.; Lin, Y.-H.; Strzalka, J.; Wang, C.; Hexemer, A.; Jaye, C.; Fischer, D. A.; Verduzco, R.; Wang, Q.; Gomez, E. D. Photovoltaic Performance of Block Copolymer Devices Is Independent of the Crystalline Texture in the Active Layer. Macromolecules 2016, 49 (12), 4599-4608.

(27) Scheiner, S. Noncovalent Forces; Springer International Publishing: Switzerland, 2015; Vol. 19.

(28) Kline, R. J.; McGehee, M. D.; Kadnikova, E. N.; Liu, J. S.; Frechet, J. M. J. Controlling the field-effect mobility of regioregular polythiophene by changing the molecular weight. Adv. Mater. 2003, 15 (18), 1519-1523.

(29) Chang, J. F.; Sirringhaus, H.; Giles, M.; Heeney, M.; McCulloch, I. Relative importance of polaron activation and disorder on charge transport in high-mobility conjugated polymer field-effect transistors. Phys. Rev. B: Condens. Matter Mater. Phys. 2007, 76 (20), na.

(30) Vakhshouri, K.; Smith, B. H.; Chan, E. P.; Wang, C.; Salleo, A.; Wang, C.; Hexemer, A.; Gomez, E. D. Signatures of Intracrystallite and Intercrystallite Limitations of Charge Transport in Polythiophenes. Macromolecules 2016, 49 (19), 7359-7369.

(31) Hamadani, B. H.; Gundlach, D. J.; McCulloch, I.; Heeney, M. Undoped polythiophene field-effect transistors with mobility of $1 \mathrm{~cm} 2$ V-1 s-1. Appl. Phys. Lett. 2007, 91 (24), 243512.

(32) McCulloch, I.; Heeney, M.; Bailey, C.; Genevicius, K.; Macdonald, I.; Shkunov, M.; Sparrowe, D.; Tierney, S.; Wagner, R.; Zhang, W. M.; Chabinyc, M. L.; Kline, R. J.; McGehee, M. D.; Toney, M. F. Liquid-crystalline semiconducting polymers with high chargecarrier mobility. Nat. Mater. 2006, 5 (4), 328-333. 